\title{
Should pediatric infectious diseases physicians be proponents of probiotics?
}

\author{
Joan L Robinson MD
}

$\mathrm{P}$ robiotics are live bacteria or fungi deliberately introduced into the gastrointestinal (GI) tract in an attempt to prevent or treat a disease state. Probiotics are believed to work using three mechanisms $(1,2)$. The first is a direct antimicrobial effect. Probiotic strains are postulated to 'crowd out' pathogenic GI flora and to compete with them for elements, such as iron, to act as 'decoy binding sites', such that pathogens bind to them rather than to mucosal surfaces and to produce antibacterial products including bacteriocins (bacterial toxins that inhibit other bacteria), hydrogen peroxide and organic acids. The second mechanism is alteration of the GI mucosal barrier. Colonization of probiotic strains may prevent pathogens from damaging the mucosa and invading. The third mechanism is through effects on mucosal immunity, leading to nonspecific humoral immune responses, production of protective cytokines and induction of regulatory $\mathrm{T}$ cells, which have an anti-inflammatory effect.

Despite decades of use, the efficacy of probiotics for many indications remains unclear. Reasons for this include:

1. There are $>100$ products worldwide marketed as probiotics. Clinical trials have used myriad products and formulations (based primarily on local availability) and a wide range of doses. Natural health products, including probiotics, are typically not required to meet the same quality standards as pharmaceuticals; therefore, it is possible that the products used in different trials were not uniform, even if they came from the same manufacturer. It is also possible that some formulations did not contain live organisms at the time of ingestion or that probiotic organisms may have been killed by antibiotics given simultaneously (although some believe that even 'dead' probiotics may have some efficacy). In summary, negative trials for any indication do not rule out efficacy of other probiotic regimens for that indication.

2. The United States Food and Drug Administration does not have a definition for 'probiotics' or recognize them as a unique product, and currently requires the same rigour of clinical trial for them as for any new pharmaceutical, including starting with phase I trials. This has led to a marked paucity of probiotic clinical trials in the United States.

3. Clinical trials of probiotics as prophylaxis requires a large sample size because such trials target adverse events that occur for a minority of patients and probiotics are never anticipated to have $100 \%$ efficacy.

There is overwhelming evidence that probiotics prevent necrotizing enterocolitis and decrease mortality in preterm infants with a birth weight $>1000 \mathrm{~g}$ (3). Due to safety concerns, there are few clinical trials involving smaller infants, Two recent trials involving 1200 infants with a birth weight of $<1000 \mathrm{~g}$ showed a trend toward a decrease in the incidence of necrotizing enterocolitis (risk ratio 0.76 [95\% CI 0.37 to 1.58 ]) (3). I calculate a number needed to treat to prevent one case (NNTT) of 47 (95\% CI 24 to 693) from these two trials. It is conceivable that probiotics are more effective in newborns than in other populations because their lack of diverse GI flora makes it more likely that a probiotic strain will find a hospitable niche.

The following are the main indications related to pediatric infectious diseases for which probiotics are considered.

Primary or secondary prevention of Clostridium difficile-associated diarrhea

Three recent systematic reviews of primarily adult trials concluded that there is moderate quality evidence that probiotics are effective for primary prevention of $\mathrm{C}$ difficile-associated diarrhea (CDAD) (4). Efficacy was $60 \%$ in three pediatric trials $(n=605)$ versus $64 \%$ in 19 adult trials $(n=3551)$ in the 2013 Cochrane review (5), with the incidence of CDAD decreasing from $5.5 \%$ to $2.0 \%$. The NNTT in all ages was 29 (95\% CI 22 to 43). Separating out the three pediatric trials (with a mix of inpatients and outpatients) (6-8), I calculate a NNTT that is also 29 (95\% CI 15 to 250).

In an adult hospital, when the practice of automatically prescribing the probiotic Saccharomyces boulardii with broad-spectrum antibiotics ended, the incidence of hospital-onset CDAD remained at approximately one per 1000 patient days, suggesting a lack of utility for routine prophylactic probiotics in a centre with a low incidence of CDAD (9).

My conclusion is that one should consider prophylactic probiotics only in medically fragile children prescribed antibiotics commonly associated with CDAD (quinolones, clindamycin, cephalosporins and carbapenems) while admitted to a hospital with a high incidence of CDAD.

Experts are less optimistic that probiotics can prevent recurrent CDAD because GI flora is so disrupted in patients with recurrent CDAD that probiotics may be ineffective (4). Two small trials involving adults yielded discordant results (4). Currently, probiotics are not indicated for secondary prevention of CDAD.

Prevention of antibiotic-associated diarrhea

A 2011 Cochrane review described a 48\% reduction in pediatric antibiotic-associated diarrhea (AAD) with probiotics (the incidence fell from $19 \%$ to $8 \%$ ) with a NNTT of 8 (95\% CI 6 to 10) but rated the evidence as low quality (10). There was a trend toward higher efficacy in those receiving $>5$ billion colony-forming units/day than in those receiving lower doses. There appears to have been only one subsequent published randomized controlled trial yielding similar findings (11).

AAD trials use variable definitions of diarrhea because there is no well-validated diarrhea scoring system that is robust in all age groups, resulting in inconsistent primary outcomes. Many had large numbers of children lost to follow-up.

Because most cases of AAD are brief and do not result in medical visits, it is my opinion that probiotics should not be prescribed for prevention of $\mathrm{AAD}$, although one may consider use in a child with a history of bothersome AAD. My opinion will change if higher-quality trials demonstrate a lower NNTT.

University of Alberta and Stollery Children's Hospital, Edmonton, Alberta

Correspondence: Dr Joan L Robinson, University of Alberta and Stollery Children's Hospital, 3-556 ECHA 11405-87 Avenue, Edmonton, Alberta T6G 1C9. Telephone 780-248-5540, fax 888-790-1176, e-mail jr3@ualberta.ca; 


\section{Treatment of acute diarrhea}

A 2010 Cochrane review of primarily pediatric inpatient trials of presumed infectious diarrhea of any etiology reported that probiotics decrease the duration of diarrhea by $25 \mathrm{~h}$ (95\% CI $16 \mathrm{~h}$ to $34 \mathrm{~h}$ ) ( 35 trials), decrease the absolute number of stools on day 2 by 0.80 (95\% CI 0.45 to 1.14 ) (20 trials) and decrease the risk of having diarrhea for $>4$ days by $60 \%$ (29 trials) (12). I calculate a NNTT of 4 (95\% CI 3.4 to 4.4 ) for the latter outcome. Four subsequent trials reported similar results (13-16), while a fifth reported no efficacy in Indonesian outpatients (17). A 2013 systematic review included only trials conducted in children $<5$ years of age and excluded trials limited to a specific pathogen (such as rotavirus) (18). They found eight lowto moderate-quality trials and reported that probiotics reduce diarrhea duration by $14.0 \%$ (95\% CI $3.8 \%$ to $24.2 \%$ ) and reduce stool frequency on day 2 by $13.1 \%$ ( $95 \%$ CI $0.8 \%$ to $25.3 \%$ ), but do not decrease hospitalizations. A recent review contended that the need for subsequent health care visits within seven days is a more relevant outcome than the duration or severity of diarrhea, but found no trials that reported this outcome (19).

All the same caveats related to defining diarrhea mentioned for the AAD trials also apply to these trials. In conclusion, I would not recommend probiotics for treatment of acute diarrhea unless greater benefit is demonstrated in future trials; however, others may interpret the data differently.

\section{Prevention of viral infections}

A 2015 Cochrane review included 13 trials, of which eight involved children. Many involved administration of probiotic drinks for several months to children in child care centres (20). This review concluded that there is low-quality evidence that probiotics prevent upper respiratory tract infections (URTIs), decrease the duration of URTIs and prevent the use of antibiotics and absenteeism. The effect size of the five pediatric trials that reported the outcome of "minimum one URTI" was 0.43 (95\% CI 0.29 to 0.63 ), which is a medium effect. The pediatric trials included a day care trial (21) and a hospital trial by a group in Croatia (22), both demonstrating prevention of URTIs with Lactobacillus GG (the NNTT was 30 in the hospital study). Only the hospital study demonstrated efficacy for prevention of GI infections

\section{REFERENCES}

1. Patel R, DuPont HL. New approaches for bacteriotherapy:

Prebiotics, new-generation probiotics, and synbiotics. Clin Infect Dis 2015;60:S108-21.

2. MacFarland LV. From yaks to yogurt: The history, development and current use of probiotics. Clin Infect Dis 2015;60:85-90.

3. AlFaleh K, Anabrees J. Probiotics for prevention of necrotizing enterocolitis in preterm infants. Cochrane Database Syst Rev 2014;(4):CD005496.

4. Evans CT, Johnson S. Prevention of Clostridium difficile infection with probiotics. Clin Infect Dis 2015;60:S122-8.

5. Goldenberg JZ, Ma SS, Saxton JD, et al. Probiotics for the prevention of Clostridium difficile-associated diarrhea in adults and children. Cochrane Database Syst Rev 2013;5:CD006095.

6. Arvola T, Laiho K, Torkkeli S, et al. Prophylactic Lactobacillus GG reduces antibiotic-associated diarrhea in children with respiratory infections: a randomized study. Pediatrics 1999;104:e64.

7. Kotowska M, Albrecht P, Szajewska H. Saccharomyces boulardii in the prevention of antibiotic-associated diarrhoea in children: A randomized double-blind placebo-controlled trial. Aliment Pharmacol Ther 2005;21:583-90

8. Ruszczyński M, Radzikowski A, Szajewska H. Clinical trial: Effectiveness of Lactobacillus rhamnosus (strains E/N, Oxy and Pen) in the prevention of antibiotic-associated diarrhoea in children. Aliment Pharmacol Ther 2008;28:154-61

9. Flatley EA, Wilde AM, Nailor MD. Saccharomyces boulardii for the prevention of hospital onset Clostridium difficile infection. J Gastrointestin Liver Dis 2015;24:21-4.

10. Johnston BC, Goldenberg JZ, Vandvik PO, Sun X, Guyatt GH. Probiotics for the prevention of pediatric antibiotic-associated diarrhea. Cochrane Database Syst Rev 2011;(11):CD004827.
$(\mathrm{NNTT}=15)$. The same group repeated both trials using Bifidobacterium animalis subspecies lactis, yielding negative results $(23,24)$. This indicates that probiotic efficacy for prevention of viral infections may be strain specific.

My opinion is that probiotics should not be used for prevention of viral infections because there is a need for higher-quality data with closer tracking of adverse events with long-term use.

\section{Prevention of ventilator-associated pneumonia}

A 2014 Cochrane review reported that there is low-quality evidence from eight trials involving 1083 adults that probiotics prevent ventilator-associated pneumonia (VAP) (25). An open-label pediatric trial from India reported a decrease in the incidence of VAP from $48 \%$ to $17 \%(\mathrm{P}<0.001)(26)$. However, the diagnosis of VAP is far from objective and often primarily based on positive airway cultures. Altering GI flora may impact airway colonization and prevent the overdiagnosis of VAP rather than actually preventing VAP (27), which may still be a worthwhile intervention! Clearly, more study is required before probiotics are recommended for prevention of VAP.

\section{Adverse effects of probiotics}

Published cases of invasive infections from probiotics are very rare, even in preterm infants (28). It appears possible that such cases may be under-reported because blood cultures are not always performed before starting antimicrobials and the volume of blood collected is often suboptimal, especially in preterm infants. Unfortunately, adverse events are often very poorly reported in clinical trials; however, no consistent clinically important adverse events have been uncovered to date. There are theoretical concerns that probiotics may increase oxygen demand in the gut mucosa, leading to ischemia in seriously ill patients, or may transfer resistance genes to other GI flora (29).

\section{CONCLUSION}

There is often pressure for physicians to prescribe probiotics because parents regard them as 'natural' and, therefore, safer than other medications. The cost of probiotics can be substantial. Pending more data, use of probiotics in children for prevention or therapy of infectious diseases should be very limited.
11. Investigating group for prevention of AAD in children with pneumonia by Clostridium butyricum and Bifidobacterium. Multicenter, randomized, controlled clinical trial on preventing antibiotic-associated diarrhea in children with pneumonia using the live Clostridium butyricum and Bifidobacterium combined powder. Zhonghua Er Ke Za Zhi 2012;50:732-6

12. Allen SJ, Martinez EG, Gregorio GV, Dans LF. Probiotics for treating acute infectious diarrhoea. Cochrane Database Syst Rev 2010;(11):CD003048.

13. İșlek A, Sayar E, Yılmaz A, Baysan BÖ, Mutlu D, Artan R. The role of Bifidobacterium lactis B94 plus inulin in the treatment of acute infectious diarrhea in children. Turk J Gastroenterol 2014;25:628-33.

14. Dinlevici EC1, Dalgic N, Guven S, et al. The effect of a multispecies synbiotic mixture on the duration of diarrhea and length of hospital stay in children with acute diarrhea in Turkey: Single blinded randomized study. Eur J Pediatr 2013;172:459-64.

15. Corrêa NB, Penna FJ, Lima FM, Nicoli JR, Filho LA. Treatment of acute diarrhea with Saccharomyces boulardii in infants. J Pediatr Gastroenterol Nutr 2011;53:497-501.

16. Francavilla R, Lionetti E, Castellaneta S, et al. Randomised clinical trial: Lactobacillus reuteri DSM 17938 vs. placebo in children with acute diarrhoea - a double-blind study. Aliment Pharmacol Ther 2012;36:363-9.

17. Hegar B, Waspada IM, Gunardi H, Vandenplas Y. A double blind randomized trial showing probiotics to be ineffective in acute diarrhea in Indonesian children. Indian J Pediatr 2015;82:410-4.

18. Applegate JA, Fischer Walker CL, Ambikapathi R, Black RE. Systematic review of probiotics for the treatment of communityacquired acute diarrhea in children. BMC Public Health 2013;13(Suppl 3):S16. 
19. Freedman SB, Pasichnyk D, Black KJ, et al; Pediatric Emergency Research Canada Gastroenteritis Study Group. Gastroenteritis Therapies in Developed Countries: Systematic Review and MetaAnalysis. PLoS One 2015;10:e0128754.

20. Hao Q, Dong BR, Wu T. Probiotics for preventing acute upper respiratory tract infections. Cochrane Database Syst Rev 2015;2:CD006895.

21. Hojsak I, Snovak N, Abdović S, Szajewska H, Misak Z, Kolacek S. Lactobacillus GG in the prevention of gastrointestinal and respiratory tract infections in children who attend day care centers: A randomized, double-blind, placebo-controlled trial. Clin Nutr 2010;29:312-6.

22. Hojsak I, Abdović S, Szajewska H, Milosević M, Krznarić Z, Kolacek S. Lactobacillus GG in the prevention of nosocomial gastrointestinal and respiratory tract infections. Pediatrics 2010;125:e1171-7.

23. Hojsak I, Močić Pavić A, Kos T, Dumančić J, Kolaček S. Bifidobacterium animalis subsp. lactis in prevention of common infections in healthy children attending day care centers Randomized, double blind, placebo-controlled study. Clin Nutr 2015;pii: S0261-5614(15)00135-1. doi: 10.1016/j.clnu.2015.05.004.
24. Hojsak I, Tokić Pivac V, Močić Pavić A, Pasini AM, Kolaček S. Bifidobacterium animalis subsp. lactis fails to prevent common infections in hospitalized children: A randomized, double-blind, placebo-controlled study. Am J Clin Nutr 2015;101:680-4.

25. Bo L, Li J, Tao T, et al. Probiotics for preventing ventilator-associated pneumonia. Cochrane Database Syst Rev 2014;(10):CD009066.

26. Banupriya B, Biswal N, Srinivasaraghavan R, Narayanan P, Mandal J. Probiotic prophylaxis to prevent ventilator associated pneumonia (VAP) in children on mechanical ventilation: An open-label randomized controlled trial. Intensive Care Med. 2015;41:677-85.

27. Saptharishi LG, Baalaaji M, Singhi SC. Ventilator-associated pneumonia or bacterial colonization of the airway, what do probiotics decrease? Intens Care Med 2015;41:1161.

28. Jenke A, Ruf EM, Hoppe T, Heldmann M,Wirth S. Bifidobacterium septicaemia in an extremely low-birthweight infant under probiotic therapy. Arch Dis Child Fetal Neonatal Ed 2012;97:F217-F218.

29. Doron S, Snydman DR. Risk and safety of probiotics. Clin Infect Dis 2015;60(Suppl 2):S129-134. 


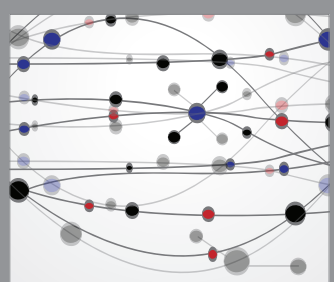

The Scientific World Journal
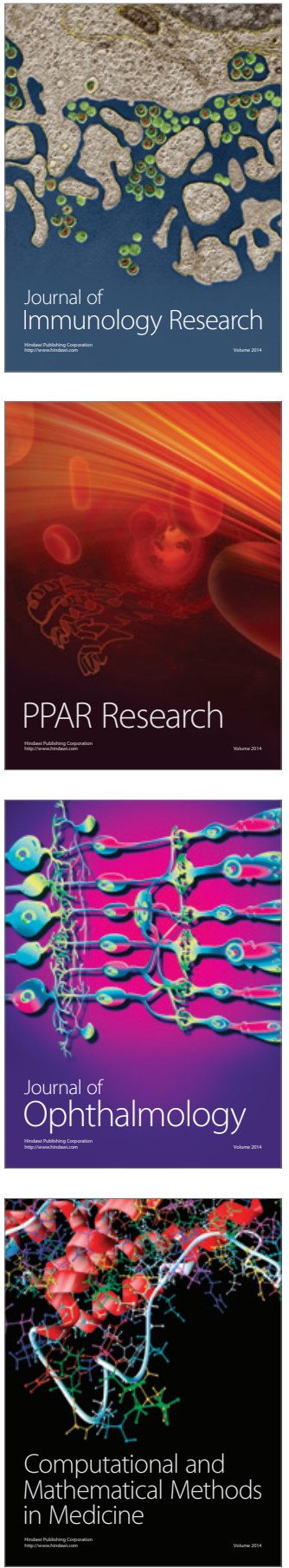

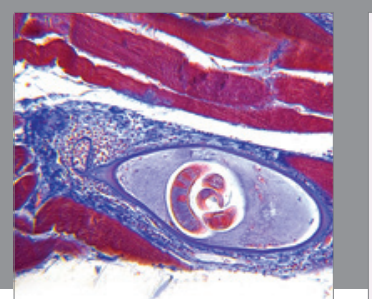

Gastroenterology Research and Practice

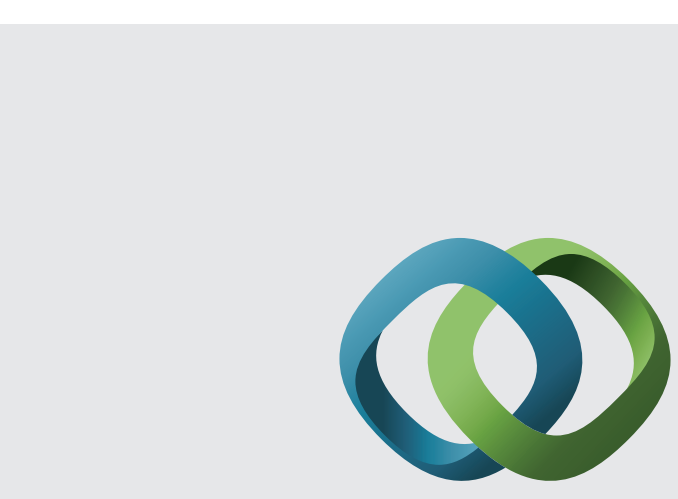

\section{Hindawi}

Submit your manuscripts at

http://www.hindawi.com
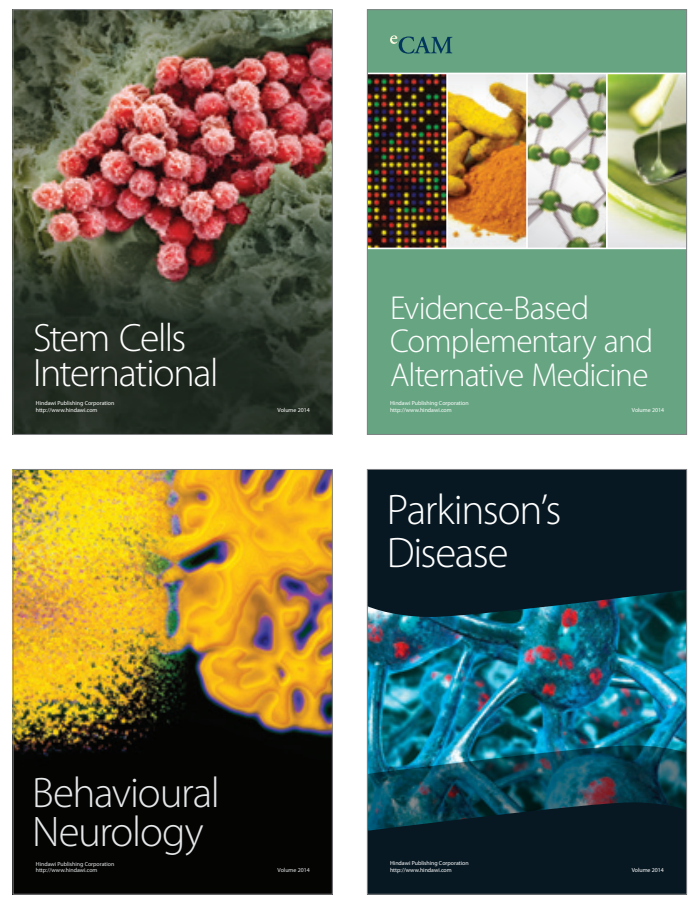
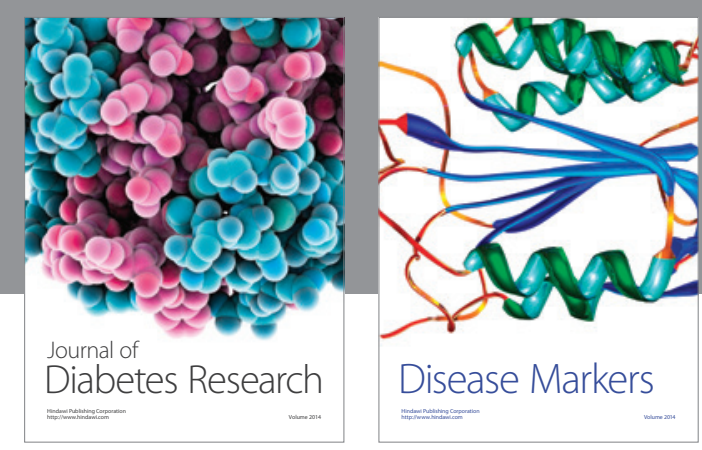

Disease Markers
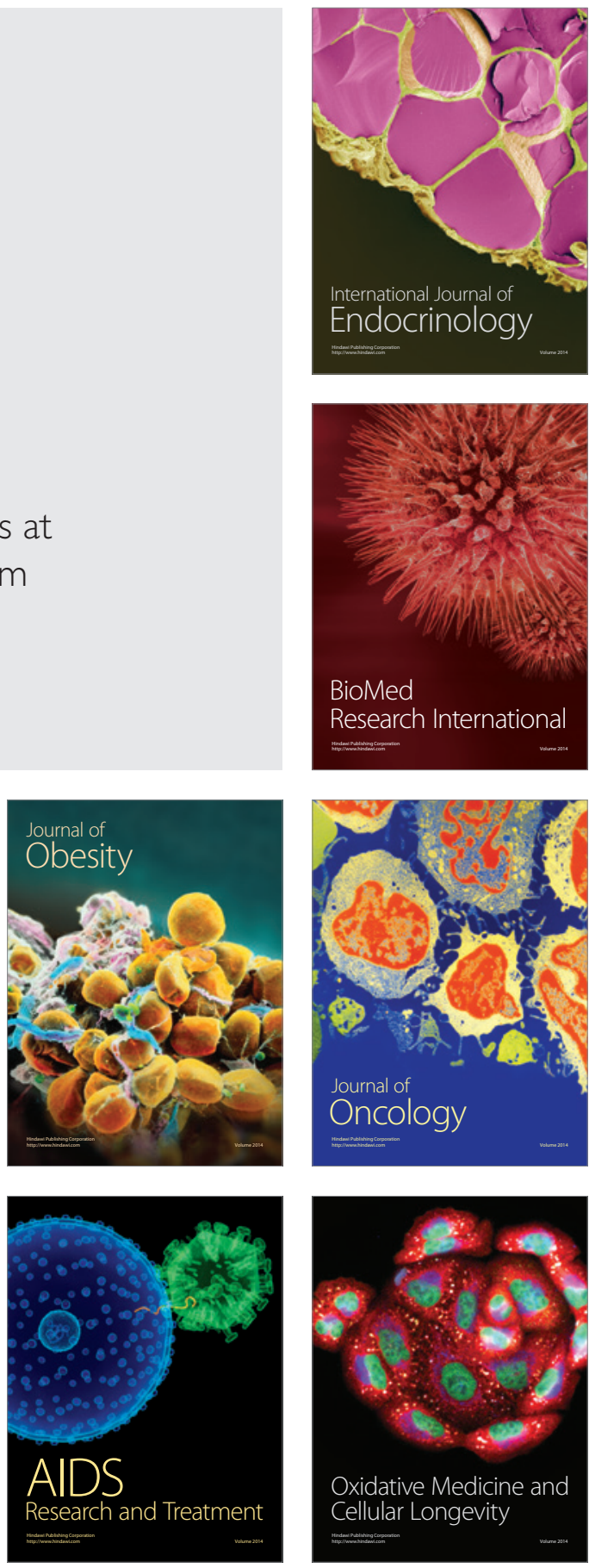